\title{
Admission Motor Strength Grade Predicts Mortality in Patients with Acute Ischemic Stroke Undergoing Mechanical Thrombectomy
}

\author{
Vishnumurthy Shushrutha Hedna ${ }^{1 *}$, Aakash N. Bodhit ${ }^{1}$, Saeed Ansari ${ }^{2}$, Adam D Falchook ${ }^{1}$, \\ Latha G. Stead ${ }^{3}$, Sharathchandra Bidari ${ }^{4}$, Brian L Hoh $^{2}$, Kenneth M Heilman ${ }^{1}$, Michael F Waters ${ }^{1,5}$ \\ ${ }^{1}$ Department of Neurology, University of Florida, Gainesville, USA $;{ }^{2}$ Department of Neurosurgery, University of Florida, Gainesville, \\ USA; ${ }^{3}$ Department of Emergency Medicine, University of Florida, Gainesville, USA; ${ }^{4}$ Department of Neuroradiology, University of \\ Florida, Gainesville, USA; ${ }^{5}$ Department of Neurosciences, University of Florida, Gainesville, USA. \\ Email: *vhedna@mail.ufl.edu
}

Received October $22^{\text {nd }}, 2012$; revised November $30^{\text {th }}, 2012$; accepted December $12^{\text {th }}, 2012$

\begin{abstract}
Background: The mortality due to mechanical thrombectomy (MT) in the acute treatment of intracranial arterial occlusions can be up to $45 \%$. The SWIFT (Solitaire FR with the Intention for Thrombectomy) and Multi MERCI (mechanical embolus removal in cerebral ischemia) trials have evaluated the safety and efficacy of MT. It may be important to determine pre-procedural factors that help predict post-intervention prognosis. We sought to determine if admission medical research council (MRC) motor strength grade along with other factors can be used as predictor of mortality after MT for acute ischemic stroke. Methods: Retrospective analysis of stroke database assessing outcomes in all 62 patients who underwent MT as an intervention for acute ischemic stroke, with or without concurrent intravenous thrombolysis was done. Five baseline variables were included in univariate and multivariate analyses to define the independent predictors of mortality during current hospitalization. The medical research council (MRC) motor grade $(0-$ 5); modified collateral flow (CS) grading (0 - 3); age; acute and chronic co-morbidities were used as the baseline variables. If motor strength grade were different in upper and lower extremities, then the lower grade was used. Age was analyzed independently as well as dichotomized using 80 as cut-off value. Relevant stroke related acute and chronic co-morbidities were given 1 point each and mean calculated. Results: In the univariate analysis, low $(0-1)$ motor strength grade $(\mathrm{OR}, 0.11 ; 95 \% \mathrm{CI}, 0.021-0.33 ; \mathrm{p}=0.001)$ and age $(\mathrm{OR}, 1.06 ; 95 \% \mathrm{CI}, 1.02-1.12 ; \mathrm{p}=0.011)$ was significantly associated with mortality. The presence of collateral flow, acute and chronic co-morbidities were not significantly associated with mortality. In the multivariate analysis, motor grade retained its statistical significance for mortality (OR, 0.09; 95\% CI, $0.01-0.32 ; \mathrm{p}=0.003)$ along with chronic co-morbidity (OR, 1.52; 95\% CI $1.05-2.43 ; \mathrm{p}=$ 0.04). Interestingly, age which is a strong predictor of favorable stroke outcome in previous studies was not statistically significant for predicting mortality $(\mathrm{OR}, 1.02 ; 95 \% \mathrm{CI}, 0.97-1.09 ; \mathrm{p}=0.32)$. Conclusions: This preliminary data suggest, admission MRC motor strength grade can serve as a robust predictor of mortality after MT in patients with acute ischemic stroke. Further validation by prospective multivariate analysis using larger sample size is needed.
\end{abstract}

Keywords: Motor Grade; Collaterals; Ischemic Stroke; Endovascular Intervention; Outcome after Stroke; Mortality after Stroke; Acute Stroke; Endovascular Treatment; Mechanical Thrombectomy

\section{Introduction}

The one month mortality from ischemic stroke ranges from $2.5 \%$ (lacunar infarction) to $78 \%$ (malignant middle cerebral artery infarction) [1,2]. The standard of care for ischemic stroke is intravenous tissue plasminogen activator (rt-PA). Patients with large vessel occlusion or tandem occlusions receiving intravenous thrombolysis are

\footnotetext{
"Corresponding author.
}

less likely to attain recanalization, with recanalization rates estimated to be $43 \%$ [3]. The recent mechanical thrombectomy (MT) strategies including SOLITAIRE FR have favorably transformed the paradigms of reperfusion approach in acute ischemic stroke [4]. However, mortality from these procedures is significant ranging from $16 \%-45 \%$ depending upon the involvement of the vessels, devices and methodology [3,5]. The outcome after MT is variable and detailed knowledge of factors 
associated with desirable or poor outcomes is therefore essential.

The NIH stroke scale (NIHSS) is a standardized neurological examination that is administered in the setting of an acute stroke to measure the patient's neurological deficit [6]. Information from this assessment is considered when deciding upon treatment options including intravenous thrombolysis and MT procedures. Motor strength assessment in NIHSS generally has higher examiner agreement than does assessment of cortical functions [7]. Clinicians also often assess motor strength in accordance with the medical research council (MRC) motor strength grade. Importantly, motor strength at the time of acute stroke has independently been demonstrated to be a strong predictor of prognosis and mortality [8]. The purpose of this study was to examine the role of admission baseline factors like age, motor strength, medical comorbidities (acute and chronic), and leptomeningeal collaterals as predictors of poor outcome after MT in acute stroke. We hypothesized that severity of motor weakness was a predictor of poor outcome in patients who underwent MT.

\section{Materials and Methods}

\subsection{Patients and Techniques}

Data for patients who ultimately underwent MT (MERCI, Penumbra, Alligator, Balloon angioplasty, Stents or combination of these procedures) for acute stroke between January 2007 and March 2011 from the stroke database were analyzed. This blinded, retrospective analysis of admission data included demographics, examination and computerized tomogram angiography (CTA) of the head and neck with contrast and perfusion. The inclusion criteria included: 1) MT with or without concurrent rt-PA, 2) Availability of brain imaging studies obtained as part of the pre-MT that were of sufficient quality to provide the basis for the analyses involved in this investigation; and 3 ) the availability of a well-documented on-site evaluation of extremity motor function that would provide the basis for the functional classification employed in this investigation. Subjects who did not receive any endovascular intervention were excluded. The final cohort was 62 subjects and this study was approved by the University of Florida Institutional Review Board. Two patients were intubated or sedated. In few patients documentation was incomplete for information like NIHSS, door to catheter time (catheter insertion for cerebral angiogram), thrombolysis in myocardial infarction (TIMI) score. Hence, these parameters were excluded in the analysis. One patient discharged to hospice, was included in the poor outcome category.

\subsection{Clinical Variables and Measurement of Outcome}

Poor outcome was defined as death (or equivalent to modified Rankin Scale of 6). The following baseline clinical variables and radiographic outcome measurements were retrospectively collected and included in this analysis: age, gender, acute co-morbidities, chronic comorbidities, motor grade, and collateral flow as assessed by CTA. All patients had CTA of the head and neck with perfusion imaging. The admission medical research council (MRC) motor strength grade was obtained as follows: $0=$ no muscle movements, $1=$ visible muscle movement but no movement at the joint, $2=$ movement at the joint but not against gravity, $3=$ movement against gravity but not against resistance, $4=$ movement against resistance but less than normal, and $5=$ normal strength; lower numbers reflecting greater weakness. If motor grade weakness was different in upper and lower extremities, then the lower score was used. For intubated patients, a motor grade of 0 was used. If motor strength grade was different in upper and lower extremities, then the lower grade was used. The baseline head CT angiogram (CTA) was interpreted and graded by two blinded interpreters as per the modified collateral flow (CS) grading system $(0-3) ; 0=$ absent, $1=\leq 50 \%, 2=$ $>50 \%$ but $<100 \%$ and $3=100 \%$ of collateral supply of the occluded MCA territory. The inter observer agreement for collateral scoring (kappa) was 0.83 . Collateral flow was also measured as a dichotomous variable as being present or not. The co-morbidities of each patient were divided into 2 groups: Acute co-morbidities comprising of acute complications in the current admission (pneumonia, sepsis, dissection, myocardial infarction, gastrointestinal bleed, renal failure and others) in each category were given 1 point each and the mean for that group was calculated. Chronic co-morbidities (HTN, DM, previous coronary artery disease, dissection, Peripheral vascular disease, dyslipidemia, recreational drug use and other contributory risk factors) were given 1 point each and mean calculated for each group.

\subsection{Statistical Analysis}

The database was interrogated by using univariate/ multivariate techniques. Few factors were dichotomized at clinically relevant cutoffs. For both univariate and multivariate analyses, the aforementioned 5 categorical and quantitative variables that were considered possibly related to outcome were selected from pretreatment and periprocedural clinical data. All analyses were performed by a biostatistician with the aid of JMP software (Version 8.0). A univariate analysis was first performed to assess the relation between individual baseline variables and the 
outcome measure (current admission mortality; $\mathrm{mRS}=6$ ). Only variables with $\mathrm{p}<0.20$ (except acute co-morbidity) in the univariate logistic regression analysis and present for at least $95 \%$ of the patients with the outcome variable were included in the multivariable logistic regression model-building process. Models were built using forward/backward stepwise logistic regression with variables entered into the model at the 0.05 significance level.

\section{Results}

Table 1 demonstrates the distribution of the different variables according to poor outcome post procedure in the current hospitalization. This table also includes same information of patients who survived this hospitalization and were discharged. The final cohort was comprised of 62 patients whose median age was $69 ; 29$ were women and 33 males. Thirty patients received IV rt-PA and mechanical thrombectomy (MT) while thirty two received MT alone. Two of them had basilar occlusion.

\subsection{Poor Outcome (Death)}

There were nineteen (29\%) total deaths; youngest patient was forty seven and oldest patient was ninety one. There were ten males and nine females. In this group, thirteen deaths $(68 \%)$ were vascular related and six $(32 \%)$ were due to medical co-morbidities (non-vascular related death). In the first group, vascular deaths can be further subdivided into 9 cases of hemorrhagic conversion with mass effect and 4 cases of edematous infarct with mass effect. Sub group mortality analysis $(\mathrm{n}=19)$ : IV rt-PA+ MT group $=7$ (5 of which were vascular related); MT only group $=12$ (7 of which were vascular related). All of these patients had a MRC strength grade of 0 - 1 at the time of admission. However, absence of collaterals was not significantly associated with poor outcome. The admission mean MRC motor grade was 0.1 and the median MRC motor grade was 0 (Zero).

\subsection{Predictors of Poor Outcome during Current Hospitalization}

Results of the univariate and multivariate model, which included 62 patients in the mortality (poor outcome) analysis, are summarized in Table 2. In the univariate analyses; Age (OR, 1.06; 95\% CI, $1.02-1.12 ; \mathrm{p}=0.011$ ), and motor grade on presentation (OR, $0.11 ; 95 \% \mathrm{CI}$, $0.021-0.33 ; p=0.001)$ reached statistical significance by the criterion mentioned in the methods and thus demonstrated strong evidence of an influence on poor outcome (also see Figure 1).

- Table 2 also compares univariate and multivariate analyses. In the multivariate analyses; the motor grade (OR, 0.09; 95\% CI, $0.01-0.32 ; \mathrm{p}=0.003$ ) was still the strongest predictor of poor outcome. Also showing significant impact on mortality was chronic co-morbidities (OR, 1.52; 95\% CI $1.05-2.43 ; \mathrm{p}=$ $0.04)$. Age was analyzed as whole and also dichotomized into age 80 and above. It was showing positive

Table 1. Relationship between baseline variables and outcome.

\begin{tabular}{ccc}
\hline & $\mathrm{m}^{-\mathrm{RS}^{*}=6 \text { (Dead) }}$ & $\mathrm{m}-\mathrm{RS}<6$ (Alive) \\
\hline Total number (62) & 19 & 43 \\
Received MT only & 11 & 22 \\
Received IV rt-PA + MT & 8 & $23 / 20$ \\
Number of males/females & $10 / 9$ & $62.2(17.5)$ \\
Mean age (SD) & $74.7(11)$ & 0.41 \\
Mean Acute co-morbidities & 0.42 & 3.14 \\
Mean Chronic co-morbidities & 4.26 & $2(2-3)$ \\
Mean MRC motor strength grade & 0.1 & 3.75 \\
Median MRC motor strength grade & 0 & $4(\mathrm{IQR}=2-5)$ \\
Mean m-RS score at discharge & 6 & 1.67 \\
Median m-RS score at discharge (IQR) & 6 & $13.67(588 / 43)$ \\
Mean collateral flow score & 1.34 & $10(191 / 19)$ \\
\hline Mean length of hospital stay (days/N of patients)
\end{tabular}

m-RS: modified Rankin scale; MRC: Medical research council; IQR: Interquartile range; SD: Std.Deviation; MT: mechanical thrombectomy. 
Table 2. Comparison of univariate and multivariate analyses on poor outcome.

\begin{tabular}{|c|c|c|}
\hline Variables & Univariate OR ( $95 \% \mathrm{CI})$; P-value & Multivariate OR (95\% CI); P-value \\
\hline Age & 1.06 (1.02 - 1.12); 0.011 & $1.02(0.97-1.09) ; 0.32$ \\
\hline Age (80-cut off) & 0.27 (0.07 - 0.98); 0.048 & $0.54(0.07-3.35) ; 0.51$ \\
\hline Acute co-morbidity & $1.003(0.49-1.84) ; 0.90$ & $0.93(0.33-2.24) ; 0.88$ \\
\hline Chronic co-morbidity & $1.19(0.96-1.52) ; 0.11$ & 1.52 (1.05 - 2.43); 0.04 \\
\hline Collaterals & 0.45 (0.17-1.05); 0.08 & $0.30(0.04-1.35) 0.12$ \\
\hline Motor grade & $0.11(0.02-0.33) ; 0.001$ & $0.095(0.013-0.32) 0.003$ \\
\hline
\end{tabular}

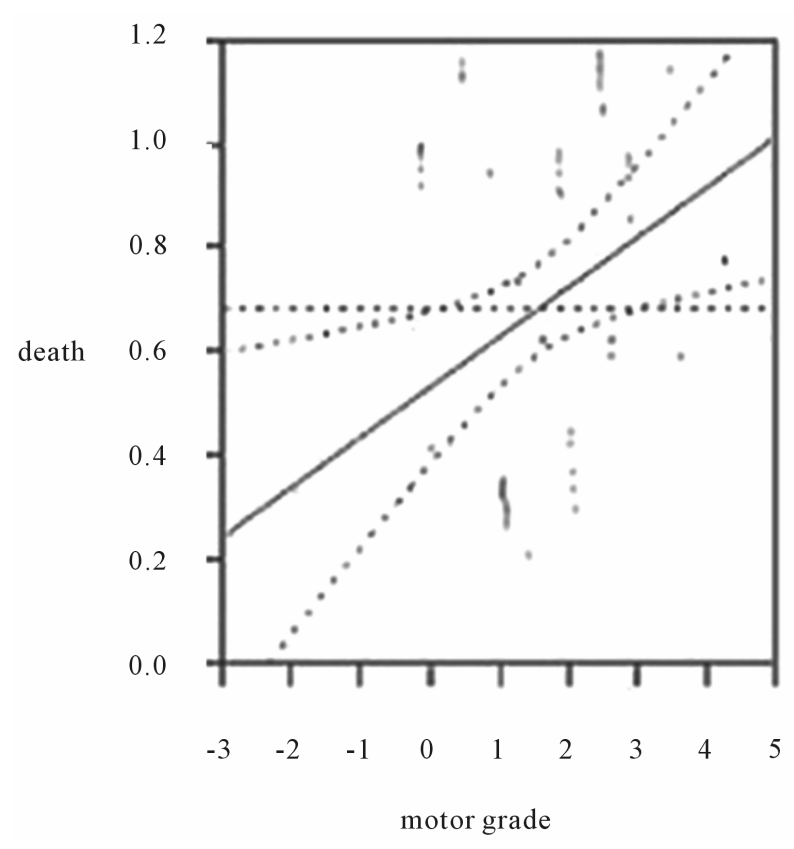

Figure 1. Influence of motor grade on poor outcome in our cohort. All of the patients who died had a MRC motor strength grade of 0 - 1 at the time of admission.

impact on poor outcome only in the univariate analysis. Age did not have any impact in the multivariate analyses (OR, 1.02; 95\% CI, $0.97-1.09 ; \mathrm{p}=0.32$ ). Acute comorbidities, Collaterals on imaging did not have any impact in both univariate and multivariate analyses. Interestingly, the motor grade on admission showed a trend to predict m-RS at discharge as shown in Tables 3 and 4.

- ANOVA test was done to see the difference in MRC scores among three categories of $\mathrm{m}$-RS (The three categories were: Category $1=\mathrm{m}-\mathrm{RS} \leq 2$; Category 2 $=\mathrm{m}-\mathrm{RS} 3-5$; Category $3=\mathrm{m}-\mathrm{RS}$ 6). We assumed MRC as a continuous variable for this analysis. ANOVA showed a statistically significant difference in MRC scores among three categories $(p<0.0001)$, with mean MRC scores for categories 1, 2 and 3 were $3.15,1.8$ and 0.1 respectively. A post-hoc analysis using Tukey's HSD test showed that there was statistically significant difference between two categories of $\mathrm{m}$-RS when the categories were compared in pairs.

- Category 1 and 3: mean difference in MRC scores was $3.04(\mathrm{p}<0.0001)$;

- Category 2 and 3: mean difference in MRC scores was $1.69(\mathrm{p}<0.0001)$;

- Category 1 and 2: mean difference in MRC scores was $1.35(p=0.001)$.

Patients with lower MRC motor grade had higher $\mathrm{m}$-RS on discharge and patients with lower m-RS on discharge had higher MRC grade on admission.

\section{Discussion}

In this small cohort of patients with mechanical thrombectomy for acute ischemic stroke, lower motor grade was a strong predictor of mortality and was directly associated with poor outcome. Patients with low admission motor strength grade of 0 or 1 in one or both extremities on one side had higher rates of mortality, while those who survived had greater motor strength.

Table 3. Comparison of motor score among different m-RS categories by ANOVA.

\begin{tabular}{cccc}
\hline m-RS & $\begin{array}{c}\text { Number of } \\
\text { patients }\end{array}$ & $\begin{array}{c}\text { Motor grade } \\
\text { (mean } \pm \text { SD) }\end{array}$ & CI \\
\hline$\leq 2$ & 13 & $3.15 \pm 1.46$ & $2.27-4.03$ \\
$3-5$ & 30 & $1.8 \pm 1.21$ & $1.34-2.25$ \\
6 & 19 & $0.10 \pm 0.31$ & $-0.04-0.25$ \\
\hline
\end{tabular}

Table 4. Comparison of motor scores among different m-RS categories by Tukey's HSD test.

\begin{tabular}{cccc}
\hline m-RS & $\begin{array}{c}\text { Difference in } \\
\text { motor score }\end{array}$ & CI & P value \\
\hline$\leq 2$ and 6 & 3.04 & $2.1-3.99$ & $<0.0001$ \\
$3-5$ and 6 & 1.69 & $0.92-2.46$ & $<0.0001$ \\
$<2$ and 3 - 5 & 1.35 & $0.48-2.22$ & 0.001 \\
\hline
\end{tabular}


Interestingly, the motor grade on admission was also able to predict $\mathrm{m}$-RS at discharge with lower MRC motor grade having higher $\mathrm{m}-\mathrm{RS}$ on discharge and patients with lower m-RS on discharge had higher MRC grade on admission. Our findings are consistent with prior studies of acute stroke treatment. Heald et al. studied patients with ischemic stroke using transcranial magnetic stimulation to assess central motor conduction time, a parameter that is thought to reflect activity in the corticospinal tract [8]. Compared to stroke patients with normal or delayed responses, stroke patients with absent responses in the thenar muscles to electromagnetic stimulation of the cortex had more profound weakness at the time of acute stroke and increased length of hospital stay. When assessed at twelve months after their stroke, they had more severe disability as assessed with the modified Rankin Scale, and higher rates of mortality. Recent studies with MRI show wallerian degeneration of the corticospinal tract can occur even in acute phase of ischemic stroke [9]. This has been associated with poor motor outcome after stroke. The factors like reactive hyperemia after MT in the infarcted tissue zone can lead to hyper-perfusion syndrome (HPS) [10]. This overabundant cerebral blood flow causes worsening of cytotoxic edema, setting of vasogenic edema, hemorrhagic conversion of the infarct and subsequent brain herniation. The mortality in our patients were largely due to these vascular pathogenesis.

Previous studies have shown factors like time of presentation, age, sex, NIHSS, mean arterial blood pressure, admission blood glucose, co-morbidities like diabetes, ICA occlusions, complete recanalization and early brain edema, symptomatic hemorrhages are independent predictors of outcome $[11,12]$.

Age, shown to be a strong predictor of mortality in previous studies [13] did not appear to have significant impact on mortality in this study. The results were same even for patients aged 80 and above.

The comorbidities did not have a huge impact on mortality in this study. Even though chronic co-morbidities did have some impact in the multivariate analyses, acute co-morbidities did not seem to have any effect on mortality.

Some previous studies have shown [14] absence of collaterals favor poor outcome [15]. Our study looking specifically at collaterals on admission CTA did not appear to have any effect on poor outcome. Common notion that successful recanalization and good collaterals equals excellent outcome may not be relevant in some patients. Flow restoration and attempted recanalization after MT can lead to two extreme outcomes. Complete recanalization may lead to restoration of blood flow to the infarcted and ischemic zone with no untoward com- plications helping the neurological status which is the ultimate goal. Other extreme is, variable distal patency and perfusion/reperfusion leading to a higher infarct core hemorrhage risk and hyper-perfusion injury. Mortality is imminent when damage is extensive.

There are some limitations of the current study. This is a retrospective analysis of a small cohort. The nature of the study introduces a number of potential confounders and the size further accentuates such bias and limits generalizability of the findings. Any identification of clinical outcome predictors needs further validation by prospective multivariate analysis using larger sample size. Another limitation of the study revolves around the reliability of the physical exam. If the patient is sedated, intubated, lacking comprehension and showing severe neglect, then a reliable motor strength assessment can be difficult which ultimately introduces bias into the study.

\section{Conclusion}

The role of cortical synaptic transmission and damage to the descending motor pathways in the pathogenesis of post stroke hemiplegia remain as important questions for future research. Our results demonstrate the severity of post-stroke hemiplegia is a major predictor of poor prognosis. Given the poor prognosis in patients with profound post stroke hemiplegia and the correlation between hemiplegia and poor outcome after endovascular treatment, the risks of an interventional procedure must be carefully weighed against the expected outcome from no intervention.

\section{Acknowledgements}

The Publication of this article will be funded in part by the University of Florida Open-Access Publishing Fund.

\section{REFERENCES}

[1] B. Norrving, "Long-Term Prognosis after Lacunar Infarction," Lancet Neurology, Vol. 2, No. 4, 2003, pp. 238-245. doi:10.1016/S1474-4422(03)00352-1

[2] W. Hacke, S. Schwab, M. Horn, M. Spranger, M. De Georgia and R. von Kummer, "Malignant Middle Cerebral Artery Territory Infarction: Clinical Course and Prognostic Signs," Archives of Neurology, Vol. 53, No. 4, 1996, pp. 309-315.

doi:10.1001/archneur.1996.00550040037012

[3] J. H. Rha and J. L. Saver, "The Impact of Recanalization on Ischemic Stroke Outcome: A Meta-Analysis," Stroke, Vol. 38, No. 3, 2007, pp. 967-973. doi:10.1161/01.STR.0000258112.14918.24

[4] J. L. Saver, R. Jahan, E. Levy, T. G. Jovin, B. Baxter, R. G. Nogueira, W. Clark, R. Budzik and O. O. Zaidat, "Solitaire Flow Restoration Device versus the Merci Re- 
triever in Patients with Acute Ischaemic Stroke (SWIFT): A Randomised, Parallel-Group, Non-Inferiority Trial," Lancet, No. 380, No. 9849, 2012, pp. 1241-1249. doi:10.1016/S0140-6736(12)61384-1

[5] A. Bose, H. Henke, K. Alfke, W. Reith, T. E. Mayer, A. Berlis, V. Branca and S. P. Sit, "The Penumbra System: A Mechanical Device for the Treatment of Acute Stroke Due to Thromboembolism," American Journal of Neuroradiology, Vol. 29, No. 7, 2008, pp. 1409-1413. doi:10.3174/ajnr.A1110

[6] H. P. Adams Jr., P. H. Davis, E. C. Leira, K. C. Chang, B. H. Bendixen, W. R. Clarke, R. F. Woolson and M. D. Hansen, "Baseline NIH Stroke Scale Score Strongly Predicts Outcome after Stroke: A Report of the Trial of Org 10172 in Acute Stroke Treatment (TOAST)," Neurology, Vol. 53, No. 1, 1999, pp. 126-131. doi:10.1212/WNL.53.1.126

[7] L. S. Williams, E. Y. Yilmaz and A. M. Lopez-Yunez, "Retrospective Assessment of Initial Stroke Severity with the NIH Stroke Scale," Stroke, Vol. 31, No. 4, 2000, pp. 858-862. doi:10.1161/01.STR.31.4.858

[8] A. Heald, D. Bates, N. E. Cartlidge, J. M. French and S. Miller, "Longitudinal Study of Central Motor Conduction Time Following Stroke. 2. Central Motor Conduction Measured within $72 \mathrm{~h}$ after Stroke as a Predictor of Functional Outcome at 12 Months," Brain, Vol. 116, No. 6, 1993, pp. 1371-1385. doi:10.1093/brain/116.6.1371

[9] G. DeVetten, S. B. Coutts, M. D. Hill, M. Goyal, M. Eesa, B. O'Brien, A. M. Demchuk and A. Kirton, "Acute Corticospinal Tract Wallerian Degeneration Is Associated with Stroke Outcome," Stroke, Vol. 41, No. 4, 2010, pp. 751756. doi:10.1161/STROKEAHA.109.573287

[10] W. N. van Mook, R. J. Rennenberg, G. W. Schurink, R. J. van Oostenbrugge, W. H. Mess, P. A. Hofman and P. W. de Leeuw, "Cerebral Hyperperfusion Syndrome," Lancet Neurology, Vol. 4, No. 12, 2005, pp. 877-888. doi:10.1016/S1474-4422(05)70251-9

[11] The NINDS t-PA Stroke Group, "Generalized Efficacy of t-PA for Acute Stroke. Subgroup Analysis of the NINDS t-PA Stroke Trial," Stroke, Vol. 28, No. 11, 1997, pp. 2119-2125. doi:10.1161/01.STR.28.11.2119

[12] G. Tsivgoulis, M. Saqqur, V. K. Sharma, A. Y. Lao, M. D. Hill and A. V. Alexandrov, "Association of Pretreatment Blood Pressure with Tissue Plasminogen Activator-Induced Arterial Recanalization in Acute Ischemic Stroke," Stroke, Vol. 38, No. 3, 2007, pp. 961-966. doi:10.1161/01.STR.0000257314.74853.2b

[13] R. G. Nogueira, D. S. Liebeskind, G. Sung, G. Duckwiler, and W. S. Smith, "Predictors of Good Clinical Outcomes, Mortality, and Successful Revascularization in Patients with Acute Ischemic Stroke Undergoing Thrombectomy: Pooled Analysis of the Mechanical Embolus Removal in Cerebral Ischemia (MERCI) and Multi MERCI Trials," Stroke, Vol. 40, No. 12, 2009, pp. 3777-3783. doi:10.1161/STROKEAHA.109.561431

[14] C. Brekenfeld, L. Remonda, K. Nedeltchev, F. v Bredow, C. Ozdoba, R. Wiest, M. Arnold, H. P. Mattle and G. Schroth, "Endovascular Neuroradiological Treatment of Acute Ischemic Stroke: Techniques and Results in 350 Patients," Neurology Research, Vol. 27, No. 1, 2005, pp. S29-S35.

[15] I. Y. Tan, A. M. Demchuk, J. Hopyan, L. Zhang, D. Gladstone, K. Wong, M. Martin, S. P. Symons, A. J. Fox and R. I. Aviv, "CT Angiography Clot Burden Score and Collateral Score: Correlation with Clinical and Radiologic Outcomes in Acute Middle Cerebral Artery Infarct," American Journal of Neuroradiology, Vol. 30, No. 3, 2009, pp. 525-531. doi:10.3174/ajnr.A1408 